High-resolution inductive measurement of electrical resistivity and density of electromagnetically levitated liquid metal droplets

Georg Lohöfer

Citation: Review of Scientific Instruments 89, 124709 (2018); doi: 10.1063/1.5065482

View online: https://doi.org/10.1063/1.5065482

View Table of Contents: http://aip.scitation.org/toc/rsi/89/12

Published by the American Institute of Physics

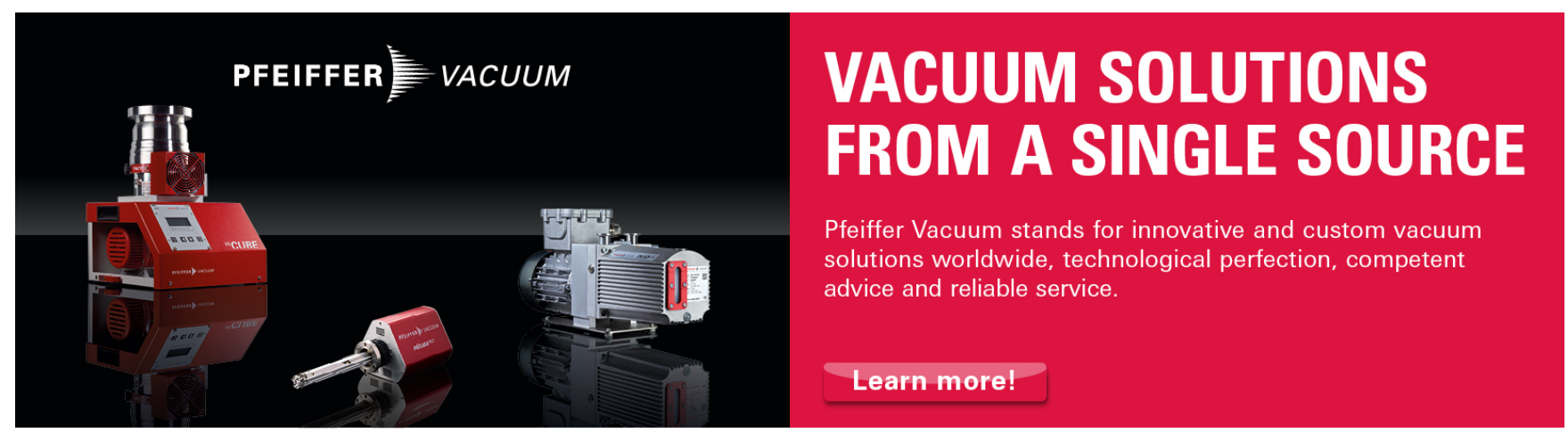




\title{
High-resolution inductive measurement of electrical resistivity and density of electromagnetically levitated liquid metal droplets
}

\author{
Georg Lohöfera) \\ Institut für Materialphysik im Weltraum, Deutsches Zentrum für Luft- und Raumfahrt (DLR), \\ 51170 Köln, Germany
}

(Received 10 October 2018; accepted 7 December 2018; published online 28 December 2018)

\begin{abstract}
A new device for the inductive measurement of electrical resistivity and density of liquid metals and semiconductors is presented. It is integrated in two electromagnetic levitation facilities operating under microgravity. As a result, the completely noninvasive handling and measuring of the metallic melt enables the extension of the accessible sample temperature range far into the undercooled liquid state below the melting point. The microgravity environment permits the undisturbed joining of the containerless inductive sample handling method by the electromagnetic levitation with the inductive sample measurement technique. The following sections explain in detail the basic principles and the technical realization of the whole measurement apparatus and present experimental data showing its high resolution resulting from the combination of microgravity, electromagnetic levitation, and inductive measurement technique. Published by AIP Publishing. https://doi.org/10.1063/1.5065482
\end{abstract}

\section{INTRODUCTION}

The relevance of the temperature $T$-dependent electrical resistivity $\rho(T)$ in technical applications is well known. During casting or in crystal growth furnaces, $\rho$ controls the melt flow under the influence of electromagnetic fields. ${ }^{1}$ Besides of this aspect, $\rho(T)$ is also a sensitive indicator for structural changes in the melt. In liquid metals, the electrical resistivity results from the scattering of the conduction electrons at the randomly distributed metal ions. It may be expected that the formation of compact structures (clusters) or a chemical short range ordering of alloy components with decreasing temperature in the melt should increase the scattering cross section for the electrons and thus also the electrical resistivity. ${ }^{2}$ This is opposite to the usually observed linear decrease in this quantity with decreasing temperature due to the reduction in the temperature-dependent density fluctuations in the melt. ${ }^{3}$ Consequently, the onset of ordering phenomena should lead to a deviation of $\rho(T)$ from the typical linear temperature dependence. ${ }^{4}$ The lower the temperature of the melt is the more pronounced this effect should be. This fact makes a measurement of $\rho$ interesting, in particular, in the undercooled melt, i.e., in the metastable liquid state below the melting temperature.

Contained in a crucible, the high temperatures and, consequently, the high reaction rates of the most investigated liquid metals with their experimental environment render a chemical inert processing a difficult task. Furthermore, since, in general, the container together with the measurement probes is inserted in a furnace, ${ }^{5}$ the thermal expansion of the melt and the crucible and the temperature dependence of the measurement equipment strongly influences the measurement procedure. These problems are bypassed if the handling of the metallic melts as well as their measurement is performed completely contactlessly. Furthermore, noninvasive processing offers the

a)E-mail: Georg.Lohoefer@dlr.de additional benefit that, due to the absence of nucleation triggering crucible walls, liquids can often be undercooled below their freezing temperatures. On the one hand, this effect may considerably enlarge the whole temperature range of the measurement because the maximum temperature of liquid metals is generally strongly limited by their exponentially growing evaporation rate with increasing temperature. On the other hand, as mentioned above, the lower the temperature of the liquid metal is the more likely a formation of structures in the melt should show up.

For the containerless handling of metallic melts, there are several levitation techniques which have to be combined with non-contact diagnostic tools for completely noninvasive measurements of thermophysical properties of liquid metals like surface tension or viscosity. ${ }^{6}$ Generally, these tools are based on optical techniques like pyrometry which applies Planck's law of radiation for temperature measurement and videometry which uses cameras for an analysis of the shape of the levitated drop. But for the latter also inductive methods can be applied. ${ }^{7}$ The contactless measurement of the electrical resistivity of a levitated sample bases naturally on inductive methods only. But also the temperature dependent volume change of a levitated liquid droplet which enables the determination of its thermal expansion can be detected inductively.

There are a lot of publications presenting experimental facilities for the contactless inductive measurement of electrical resistivities of liquid metals. Concerning the measurement process, in general, two different inductive techniques can be distinguished. The "rotating field" method $^{8-10}$ applies on a conducting sample a rotating DC magnetic field which induces eddy currents in the material. These currents together with the external circulating magnetic field generate a torque on the sample proportional to its electrical conductivity. This mechanical torque, the accurate determination of which is not a trivial task for a liquid sample, is then the actual measurement quantity. The "AC field" method ${ }^{5,11-14}$ applies on a conducting 
sample a steady radio frequency (RF) magnetic field generated by a RF coil current. The accompanying induced RF electric field drives eddy currents in the material depending on its electrical resistivity. These eddy currents in turn generate an RF magnetization field, the accompanying induced RF electric field of which reacts by driving an additional coil current, the strength and phase shift (relative to that of the applied voltage) of which has to be measured. The second method has the advantage that the measurement of pure electrical quantities is, in general, much easier than that of mechanical ones.

Concerning the handling of hot liquid metals, the experimental facilities described in Refs. 5, 8, and 9 still use crucibles to contain the melt whereas those described in Refs. 10-14 apply containerless levitation techniques for a complete contactless processing of the liquid. Concerning the requirement to reach also the undercooled state of the liquid metal-see above - only the last group is of interest.

The experimental facilities of Rhim and Ishikawa ${ }^{10}$ and Ruston et al. ${ }^{13}$ use the "electrostatic levitation" technique. Here the metal is electrically charged and lifted against gravity by a high voltage $(\approx 10 \mathrm{kV})$ electrostatic field in a vacuum environment. Besides of the complicated active controlling of the sample position between the field generating electrodes, this method is applicable for a few low vaporizing metals only because, when heated and melted by a laser, the sample evaporates material and herewith also charges from its surface.

The "aerodynamic levitation" technique, used by Enderby et al. ${ }^{11}$ and Skinner and Barnes, ${ }^{12}$ applies a chemically inert gas stream to lift the sample. Its heating and melting by a strong laser from above against the convective cooling of the gas flow from below leads, however, often to strong temperature gradients in the specimen. Furthermore, as shown in Ref. 12, the great distance between the sample and the surrounding induction (measurement) coil, necessary to prevent a heating and thus a resistance change of the coil windings, requires a relatively high measurement data resolution to detect sample resistance changes with varying sample temperatures.

"Electromagnetic levitation" is another notably simple and robust technique for the containerless handling of electrically well conducting liquids. ${ }^{15,16}$ By application of alternating magnetic fields of high frequency $(\approx 300 \mathrm{kHz})$, electromagnetic levitation stably positions and heats metallic samples without external contact by inductive means only. For the inductive resistivity measurement, the RF magnetic levitation field has to be superposed by the RF magnetic measurement field where each of these fields is generated by dedicated currents in densely arranged coils. Evidently, one of the greatest problems in this technique is caused by the mutual inductive interaction between the levitation field and the measurement field. To prevent this trouble, the very complex facility described in Ref. 14 used additional compensation transformers between the levitation and measurement circuit. Nevertheless, there were still residual voltages in the measurement circuit induced from the levitation field which could severely disturb the signals from the sample. This problem was circumvented by periodically interrupting the levitation current for a few milliseconds. Within this time which was too short to impact the position of the sample, the electrical resistivity measurement could be performed undisturbed. Another problem in evaluating the measurement data consisted in the determination of the exact shape of the levitated liquid sample which was deformed by its weight and the external electromagnetic force field.

All of these complications are almost completely removed when electromagnetic levitation and inductive measurement are performed in the largely forceless, the so called "microgravity" $(\mu \mathrm{g})$ environment. This misleading but adopted expression means that experiments are performed in free fall either onboard of aircrafts or rockets during parabolic flights or onboard of a spacecraft orbiting around the earth. Under these conditions, the lifting force can strongly be reduced, and handling and measurement of the sample can be performed independently by two superposed magnetic fields. To profit from the advantages of the microgravity, the "Institute of Materials Physics in Space" of the "German Aerospace Center" [Deutsches Zentrum für Luft- und Raumfahrt (DLR)] operates the "TEMPUS" microgravity levitation facilities for short time experiments $(\approx 20 \mathrm{~s})$ during parabolic flights on board of aircrafts and the "European Space Agency" (ESA) manages the "International Space Station (ISS)/EML" facility on board of the "International Space Station" (ISS) ${ }^{17}$ for long time experiments. Both facilities employ the same working principle and, although originally build to measure other thermophysical sample properties, ${ }^{18,19}$ they are perfectly suited also for the inductive measurement of electrical resistivity and density of metallic melts. For these purposes, they have been upgraded by an additional so called "Sample Coupling Electronics" (SCE). First attempts for an inductive measurement of electrical resistivities in $\mu \mathrm{g}$ have already been performed in 1997 with the TEMPUS/Spacelab levitation facility, a precursor of the ISS/EML facility, on board of Spacelab. ${ }^{20,21}$ However, due to the lack of a dedicated measurement electronics and the need to use the "housekeeping" data of the facility, the accuracy and resolution of the measurement results were relatively poor.

In the following, we report on the working principle of the $\mu \mathrm{g}$ electromagnetic levitation facilities and their capability for the containerless, inductive measurement method. Furthermore, we describe in detail the procedure to measure the impedance of the processed sample with the help of the SCE measurement device and present the evaluation process to determine electrical resistivity and density of the containerlessly levitated samples from this measurement quantity.

\section{ELECTROMAGNETIC LEVITATION}

Electromagnetic levitation uses a high frequency alternating magnetic field $\mathbf{B}(t)=\mathbf{B}_{0} \sin (\omega t)$ generated by alternating current carrying coil windings for the containerless lifting and heating of metallic melts. The RF magnetic field induces eddy currents in the sample. On the one hand, these currents heat the sample due to ohmic losses. On the other hand, their interaction with the original magnetic field produces Lorentz forces which point locally in a direction perpendicular to the current and the field; see Fig. 1. 


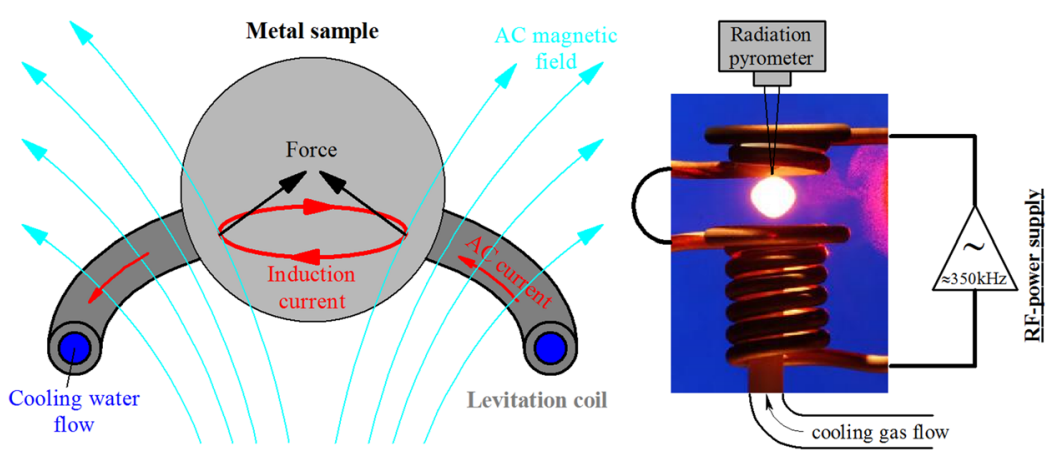

FIG. 1. Principle of electromagnetic levitation (left). The alternating magnetic levitation field induces eddy currents in the sample which, together with the field, generate Lorentz forces that support the sample against gravity. This is demonstrated in the picture of a hot liquid, levitated metal sample (right) enclosed by the alternating current carrying levitation coil on ground. The coil consists of a water cooled copper tube. The upper counter windings prevent the lateral escape of the sample. Added is the schematic of the location of the pyrometer for the contactless sample temperature measurement and the $\mathrm{He} / \mathrm{Ar}$ gas flow system for sample cooling.
In a first order approximation, the total force exerted by a weakly divergent magnetic field on a tiny metallic sphere is proportional to 22,16

$$
\mathbf{F} \propto-\mathbf{B}_{0} \cdot \nabla \mathbf{B}_{0},
$$

and the total power dissipated in it is proportional to

$$
P \propto \mathbf{B}_{0}^{2} .
$$

On ground, the diverging electromagnetic levitation field is generally used to support metallic samples of about $1 \mathrm{~g}$ mass and $5 \mathrm{~mm}$ diameter against gravity. On the one hand, electromagnetic levitation provides a technically very simple and stable containerless positioning method of metallic melts. On the other hand, however, the high magnetic force field, necessary to lift the metal against gravity, prevents very often an undisturbed measurement of thermophysical properties on a levitated liquid droplet because

- the sample deforms under the impact of the external electromagnetic forces and its weight which is clearly visible in the picture of Fig. 1.

- this part of the field which enters the droplet generates turbulent fluid flows in it which result in a strong stirring of the melt and in unsteady disturbances (oscillations) of its shape. ${ }^{23,24}$

- the strong, divergent RF magnetic levitation field interacts with the electrical circuit providing the superposed RF magnetic measurement field for the inductive determination of the electrical sample resistivity. ${ }^{14}$

- according to Eqs. (1) and (2), lifting and heating of a sample are strongly coupled so that, in general, a temperature control (reduction) has to be established by a stream of chemically inert helium or argon cooling gas blowing against the droplet.

All of these problems are strongly reduced if electromagnetic levitation is performed under microgravity conditions.

\section{A. Electromagnetic levitation under microgravity}

Due to the absence of weight in a microgravity environment, the alone remaining task of the electromagnetic positioning field consists in the containerless confinement of the metallic droplet at a certain place against external residual forces. For this purpose, the $\mu \mathrm{g}$ electromagnetic levitation facilities ISS/EML and TEMPUS use a symmetric magnetic quadrupole field produced by two equal, parallel, and coaxial circular RF currents of the same strength but opposite directions. According to Eq. (1), this so-called "positioning field" repels a displaced weightless metallic sphere to its center between the two coils where the field strength is the weakest, see Fig. 2 left, and thus, according to Eq. (2), also the heating of the sample.

Since, moreover, the residual accelerations under microgravity, which have to be compensated by the positioning field, are relatively low $\left(<10^{-2} \mathrm{~g}_{0}\right)$, the remaining magnetic field strength around a droplet can be decreased in the microgravity environment to values that are considerably weaker than those necessary to lift the same sample against its weight in $1 \mathrm{~g}$. As a result, the disadvantages of electromagnetic levitation in earth-bound laboratories like shape deformation and surface oscillations of the levitated melt are strongly reduced. But also the necessity to control (reduce) the sample temperature by an additional cooling gas flow does no longer exist.

On the other hand, this means that for an efficient inductive heating and melting of the sample, an additional, in the neighborhood of the sample, widely homogeneous RF magnetic dipole field, generated by two parallel and coaxial circular $\mathrm{RF}$ currents of the same strength and in the same directions, has to be superposed to the quadrupole field. The homogeneity has the additional advantage that, according to Eq. (1), this "heating field," though it squeezes the liquid droplet, does not however apply a significant total (center of gravity) force on it so that positioning and heating of the specimen can almost independently be performed by the dedicated fields.
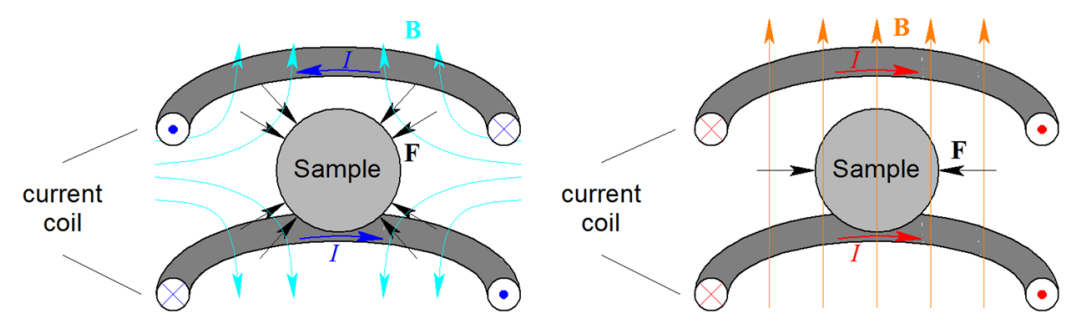

FIG. 2. Sketch of a sample in the center of two circular coils. A current in the opposite direction through the upper and lower coil (left) generates a magnetic quadrupole field for the positioning of the sample in its center. A current in the same direction through the upper and lower coil (right) generates a magnetic dipole field for the efficient heating of the sample. 

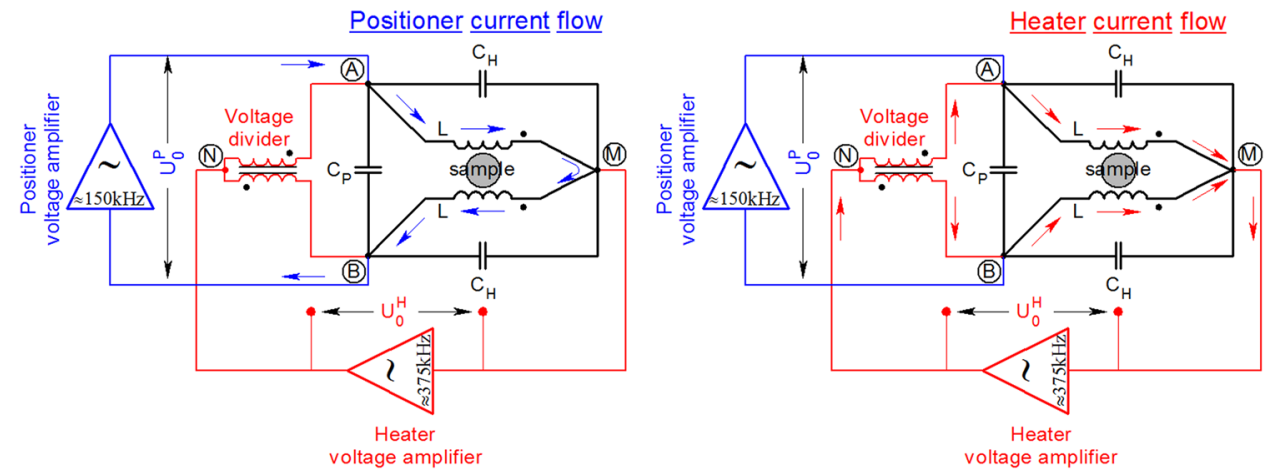

FIG. 3. Two illustrations of the electrical "Supos" circuit, applied in the ISS/EML and TEMPUS microgravity electromagnetic levitation facilities, showing the different flows of the "positioner" and "heater" currents. The RF current maintained by the positioner voltage amplifier (arrows in left diagram) flows in the opposite direction through the upper and lower coil windings thereby generating a magnetic quadrupole field; see Fig. 2 left. The RF current maintained by the heater voltage amplifier (arrows in right diagram) flows in the same direction through the upper and lower coil windings thereby generating a magnetic dipole field; see Fig. 2 right.

As already indicated in Fig. 2, both magnetic field shapes can, in principle, be created by the same coils in which the corresponding field generating currents are superposed. The electrical circuit which realizes this so called "Supos" technique $^{25}$ in the ISS/EML and TEMPUS $\mu$ g levitation facilities is schematically shown in Fig. 3.

Evidently, due to the symmetry of the currents and the resulting magnetic fields in the Supos circuit, which of course presupposes largely identical inductivities $L$ and capacities $C_{H}$ in the combined resonant circuits of Fig. 3 , the positioner current flow does not influence the voltage between the points $N$ and $M$, and the heater current flow does not influence the voltage between the points $A$ and $B$. Another benefit of this property consists in the possibility to use the heating field also for inductive measurements of electrical sample properties because

- there is no mutual interference between the heater- and positioner voltage amplifiers.

- due to the carefully designed and manufactured coils of the above mentioned $\mu \mathrm{g}$ levitation facilities, the magnetic dipole (measurement) field is largely homogeneous and thus simple and well defined in the neighborhood of the droplet.

- the forces of the magnetic dipole (measurement) field applied on the liquid sample during the inductive measurement process are too low to significantly deform the simple and well-defined spherical shape of the metallic droplet, see Sec. V A.

\section{MEASUREMENT SETUP}

\section{A. Electrical wiring diagram}

According to the right diagram of Fig. 3, the total electric heating and measurement circuit consists of two parallel resonant circuits each of which is composed of one (upper or lower) coil $L$, one capacitor $C_{H}$, and the metal sample which is inductively coupled to both of it. The schematics of the measurement circuit in which, according to Ref. 26, the (complex) sample impedance $\tilde{Z}_{S}(\omega, a, \rho)$ of the inductively coupled metal is connected in series to the coil impedance is shown in Fig. 4.
Evidently, the total heating circuit can be considered as one single parallel oscillating circuit with a total complex capacitive impedance $\tilde{Z}_{c a p}:=1 / 2 \tilde{Z}_{C}$ $=1 / 2\left(R_{C}+1 / i \omega C_{H}\right)$, where $R_{C}$ and $C_{H}$ denote the resistance and the capacitance of either of the two identical heater capacitors, respectively, and a total complex inductive impedance $\tilde{Z}_{\text {ind }}:=1 / 2\left(\tilde{Z}_{L}+\tilde{Z}_{S} / 2\right)$ composed of the sample impedance $\tilde{Z}_{S}$ and the impedance $\tilde{Z}_{L}:=R_{L}+i \omega L$ of one of the two identical coils and corresponding feeds, respectively. Here, $R_{L}$ denotes its resistance, and $L$ denotes the sum of its self-inductance and mutual inductance. The tilde $\sim$ labels complex quantities, and $i:=\sqrt{-1}$ denotes the imaginary unit. Current and voltage in the circuit are assumed to oscillate in only one single sinusoidal mode of angular frequency $\omega:=2 \pi f$. According to the

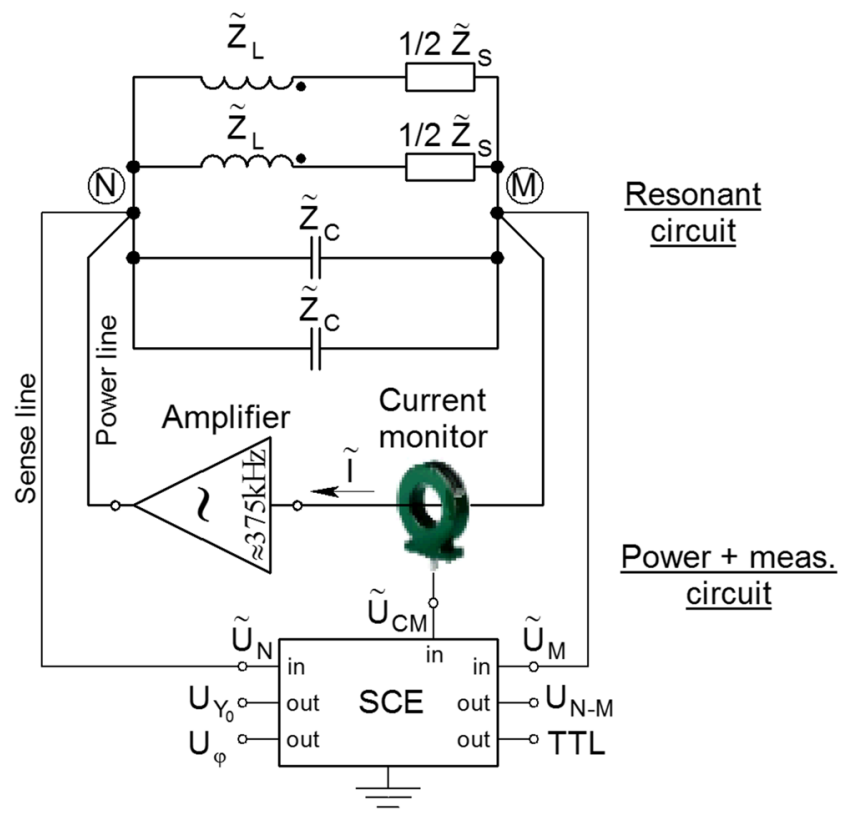

FIG. 4. Schematic wiring diagram of the ISS/EML and TEMPUS measurement circuit. It consists of the resonant heating circuit shown in Fig. 3 and the measurement setup with the inductive current monitor and the "SCE" measurement electronics which processes the RF input voltages. The influence of the here not displayed inductive voltage dividers in the sense and power line, cf. Fig. 3, is negligible. 
schematic of Fig. 3, the total complex admittance $\tilde{Y}_{\text {tot }}$, i.e., the inverse total complex impedance $\tilde{Z}_{t o t}$, of the heating circuit reads herewith

$$
\begin{aligned}
\tilde{Y}_{\text {tot }} & =\frac{1}{\tilde{Z}_{\text {tot }}}=\frac{1}{\tilde{Z}_{\text {cap }}}+\frac{1}{\tilde{Z}_{\text {ind }}} \\
& =\frac{2}{R_{C}+1 / i \omega C_{H}}+\frac{2}{R_{L}+i \omega L+\tilde{Z}_{S}(\omega, a, \rho) / 2} .
\end{aligned}
$$

Besides of the frequency $f=\omega / 2 \pi$, the total admittance of the heating circuit,

$$
\tilde{Y}_{\text {tot }}:=\tilde{I} / \tilde{U}=I_{0} / U_{0} e^{-i \varphi}
$$

is the sole externally accessible electrical quantity. It contains the measurable quantities $I_{0}, U_{0}$, and $\varphi=\varphi_{U}-\varphi_{I}$ which are the amplitude of the alternating current through the total heating circuit, the amplitude of the alternating voltage drop across the total heating circuit, and the phase shift between both, respectively. The measurement task taken by the so called "Sample Coupling Electronics" (SCE), which will be presented in Sec. IV, consists essentially in the determination of $Y_{0}:=I_{0} / U_{0}$ and of $\varphi$ out of the RF-voltages (relative to ground) from both ends of the resonant circuit and of the RF-voltage signal from the inductive current monitor which is proportional to the RF-current through the resonant circuit.

\section{B. Temperature dependency of circuit components}

The total admittance $\tilde{Y}_{t o t}$ depends sensitively on the temperatures of the copper coil and the capacitors in the measurement circuit because $R_{L}(T w)$ as well as $C_{H}(T c)$ are temperature dependent. The measurement current dissipates heat in the coil and the capacitor which is transported by the cooling water flow through the tube-like conductors; see Fig. 5.

Consequently, there is an increase in the cooling water and thus also coil temperature $T w$ along the conductor. Without going into the details of the temperature calculations, the total resistance of the coil (and the feeds) can in the steady state well be described by

$$
R_{L}(\langle T w\rangle)=R_{20} \cdot\left(1+\alpha_{\mathrm{Cu}, 20}\left(\langle T w\rangle-20^{\circ} \mathrm{C}\right)\right),
$$

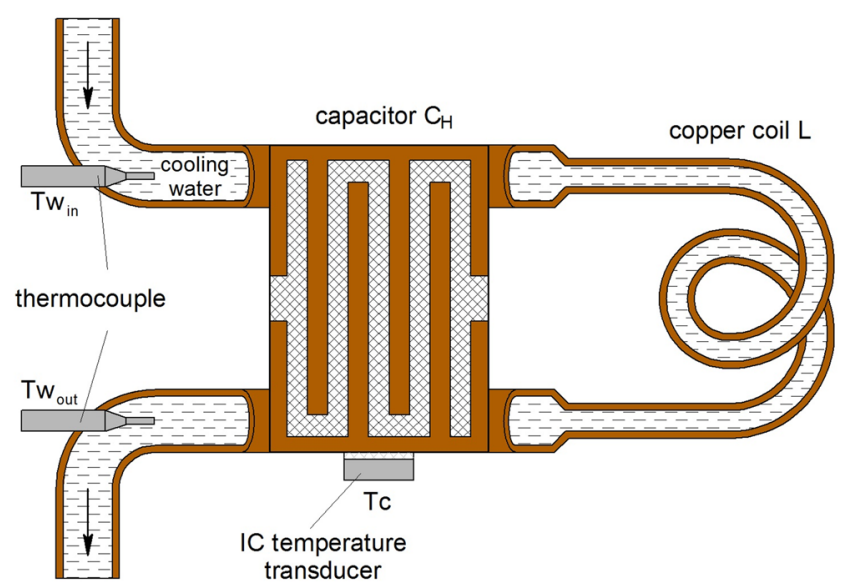

FIG. 5. Sketch of the cooling loop of one of the heating circuits, cf. Fig. 3, showing the coil $L$, the heater capacitor $C_{H}$, and the location of the temperature sensors. The temperatures of the inflowing and outflowing cooling water, i.e., $T w_{\text {in }}$ and $T w_{\text {out }}$, are measured by thermocouples. The temperature $T c$ on the outside of the capacitor is measured by a temperature transducer IC. where $R_{20}$ is the a priori unknown total resistance of the coil (and feed) at $20^{\circ} \mathrm{C}, \alpha_{\mathrm{Cu}, 20}$ is the known temperature coefficient of copper at $20^{\circ} \mathrm{C}$, and $\langle T w\rangle:=\left(T w_{\text {in }}+T w_{\text {out }}\right) / 2$ is the mean value of the inflowing and outflowing water temperatures $T w_{\text {in }}$ and $T w_{\text {out }}$, respectively, which are measured by thermocouples.

The temperature dependent capacitance of the film capacitors $C_{H}$ in the measurement circuit can empirically well be described by the first order polynomial $C_{H}(T)=C_{20}+d C / d T$. $\left(T-20^{\circ} \mathrm{C}\right)$. Due to a usually non-negligible temperature gradient between the cooling loop and the mounted capacitor block $C_{H}$, the capacitors in the measurement circuit are furnished on their surface with their own temperature transducer ICs which are largely insensitive to the external electric and magnetic fields. Furthermore, due to their extended size, there is also a temperature gradient inside of the capacitor which can be considered to be proportional to the electrical power dissipated in it. Without going into the details of the calculations, the capacity in the measurement circuit can well be described by

$$
C_{H}\left(T c, U_{0}\right)=C_{20}+d C / d T \cdot\left(T c-20^{\circ} \mathrm{C}\right)+d C / d U_{0}^{2} \cdot U_{0}^{2},
$$

where $T c$ denotes the temperature delivered by the external temperature transducer and $U_{0}$ is the amplitude of the voltage drop over the capacitor. The three coefficients $C_{20}, d C / d T$, and $d C / d U_{0}^{2}$ are a priori unknown constants and have to be determined together with $R_{20}$ and the inductance $L$ by a calibration of the measurement circuit.

\section{Theoretical basics}

The resonant circuits of the $\mu \mathrm{g}$ levitation facilities are low damped only. This means that the orders of magnitude of the terms appearing in Eq. (3) are highly different. Whereas $\omega L \approx 1 /\left(\omega C_{H}\right) \approx 1 \Omega$ there is $R_{L} \approx R_{C} \approx 0.01 \Omega$ and $\operatorname{Re}\left[Y_{\text {tot }}\right]$ $\approx\left|\operatorname{Im}\left[Y_{\text {tot }}\right]\right| \approx 0.01 \Omega^{-1}$ so that Eq. (3) can be simplified to

$$
\begin{aligned}
\frac{\tilde{Z}_{S}}{2}+ & R_{L}+i \omega L \\
= & \left(\frac{\tilde{Y}_{t o t}}{2}-\frac{1}{R_{C}+1 / i \omega C_{H}}\right)^{-1} \\
\approx & \frac{1}{\tilde{Y}_{t o t} / 2-\left(\omega C_{H}\right)^{2} R_{C}-i \omega C_{H}} \approx \frac{\operatorname{Re}\left[\tilde{Y}_{t o t} / 2\right]}{\left(\operatorname{Im}\left[\tilde{Y}_{t o t} / 2\right]-\omega C_{H}\right)^{2}} \\
& -\frac{R_{C}}{\left(1-\operatorname{Im}\left[\tilde{Y}_{t o t} / 2\right] /\left(\omega C_{H}\right)\right)^{2}}-\frac{i}{\operatorname{Im}\left[\tilde{Y}_{t o t} / 2\right]-\omega C_{H}} .
\end{aligned}
$$

After separation in the real and imaginary parts, it finally reads

$$
\begin{aligned}
& \operatorname{Re}\left[\frac{\tilde{Z}_{S}}{2}\right]+R=\frac{\operatorname{Re}\left[\tilde{Y}_{t o t} / 2\right]}{\left(\operatorname{Im}\left[\tilde{Y}_{t o t} / 2\right]-\omega C_{H}\right)^{2}}, \\
& \operatorname{Im}\left[\frac{\tilde{Z}_{S}}{2}\right]+\omega L=-\frac{1}{\operatorname{Im}\left[\tilde{Y}_{t o t} / 2\right]-\omega C_{H}},
\end{aligned}
$$

where the overall circuit resistance $R:=R_{L}+R_{C}$ has been introduced. With the knowledge of the measurement quantities $\tilde{Y}_{t o t}$ and $\omega$ and with the knowledge of the temperature dependent circuit parameters $R(\langle T w\rangle), C_{H}\left(T_{C}, U_{0}\right)$, and $L$, the sample impedance $\tilde{Z}_{S}(\omega, a, \rho)$ and thus finally the sample parameters $\rho$ and $a$ can be determined. 
For an electrically conducting sphere in a RF magnetic field, the functional dependence of $\tilde{Z}_{S}(\omega, a, \rho)$ on its specific electrical resistivity $\rho$ and radius $a$ has been derived in Ref. 26 . Especially for a nearly homogeneous RF magnetic field which is realized by the heating fields of the $\mu \mathrm{g}$ levitation facilities, this result can very well be approximated by

$$
\tilde{Z}_{S}(\omega, a, \rho)=c c \cdot \omega \cdot a^{3}\left[\frac{1}{q}-\frac{1}{q^{2}}+i\left(\frac{1}{q}-\frac{2}{3}\right)\right],
$$

under the condition, which is satisfied for the ISS/EML and TEMPUS heating circuits, that

$$
q(\omega, a, \rho):=\frac{a}{\delta}=a \sqrt{\frac{\mu_{0} \omega}{2 \rho}} \geq 3,
$$

where the dimensionless quantity $q(\omega, a, \rho)$ denotes the relation between the sample radius $a$ and the skin depth $\delta:=\sqrt{2 \rho /\left(\mu_{0} \omega\right)}$ in which $\mu_{0}$ denotes the magnetic vacuum permeability. The constant, purely coil geometry dependent factor $c c$ describes the interaction of the particular ISS/EML or TEMPUS heating coil with a sample in its center and is called "coil constant" in the following. (The product $c c \cdot \omega \cdot a^{3}$ is sometimes called "filling factor" or "coupling coefficient" in the literature.) Since $\rho$ and $a$ depend on the sample temperature $T$, all other quantities which appear in this section, except of $\mu_{0}$ and $c c$, depend on the sample temperature as well.

On the one hand, the division of the real and imaginary parts of Eq. (9), which removes the coil constant $c c$, yields

$$
\frac{\operatorname{Re}\left[\tilde{Z}_{S}\right]}{\operatorname{Im}\left[\tilde{Z}_{S}\right]}=\frac{1 / q-1 / q^{2}}{1 / q-2 / 3}<0,
$$

or solved for $1 / q$ under consideration of the inequality in (10),

$$
\frac{1}{q\left(\tilde{Z}_{S}\right)}=\frac{1}{2}\left(1-\frac{\operatorname{Re}\left[\tilde{Z}_{S}\right]}{\operatorname{Im}\left[\tilde{Z}_{S}\right]}\right)\left(1-\sqrt{1+\frac{8}{3} \frac{\operatorname{Re}\left[\tilde{Z}_{S}\right] / \operatorname{Im}\left[\tilde{Z}_{S}\right]}{\left(1-\operatorname{Re}\left[\tilde{Z}_{S}\right] / \operatorname{Im}\left[\tilde{Z}_{S}\right]\right)^{2}}}\right) \text {. }
$$

On the other hand, Eq. (10) results in

$$
\frac{\rho(T)}{a^{2}(T)}=\frac{\mu_{0} \omega(T)}{2} \frac{1}{q^{2}\left(\tilde{Z}_{S}(T)\right)} .
$$

This shows that a measurement of $\operatorname{Re}\left[\tilde{Z}_{S}\right] / \operatorname{Im}\left[\tilde{Z}_{S}\right]$ via Eqs. (7) and (8), after the circuit parameters have been determined, enables together with the knowledge of the frequency $f=\omega / 2 \pi$ the determination of the composed sample parameter $\rho / a^{2}$ via Eqs. (12) and (13).

Rearranged, the imaginary part of Eq. (9) results in

$$
c c^{\frac{1}{3}} \cdot a(T)=\left(\frac{\operatorname{Im}\left[\tilde{Z}_{S}(T)\right] / \omega(T)}{1 / q\left(\tilde{Z}_{S}(T)\right)-2 / 3}\right)^{\frac{1}{3}},
$$

and the insertion of $a$ in Eq. (13) in

$$
c c^{\frac{2}{3}} \cdot \rho(T)=\frac{\mu_{0} \omega(T)}{2} \frac{1}{q^{2}\left(\tilde{Z}_{S}(T)\right)}\left(\frac{\operatorname{Im}\left[\tilde{Z}_{S}(T)\right] / \omega(T)}{1 / q\left(\tilde{Z}_{S}(T)\right)-2 / 3}\right)^{\frac{2}{3}} .
$$

Equations (14) and (15) represent the central formulas for the determination of the sample radius $a(T)$ and the sample resistivity $\rho(T)$. On the right-hand side of both equations there appear only quantities which can be related to the measurement values $Y_{0}:=I_{0} / U_{0}$ and $\varphi$. Since in $\mu \mathrm{g}$ the liquid droplet is nearly spherical during the measurement process, its mass density is obtained by

$$
\rho_{m}(T)=\frac{m}{4 / 3 \pi a^{3}(T)},
$$

where $m$ denotes the (previously measured) sample mass.

\section{THE SCE MEASUREMENT ELECTRONICS}

To evaluate the RF-voltage signals of the measurement circuit, both $\mu \mathrm{g}$ levitation facilities are equipped with the so called "Sample coupling electronics" (SCE). As already shown in Fig. 4, the RF voltages $\tilde{U}_{N}$ and $\tilde{U}_{M}$ (relative to ground) from both ends of the measurement circuit as well as the RF voltage signal from the current monitor $\tilde{U}_{C M}$ which is proportional to the current $\tilde{I}$ through the circuit are routed to the SCE. Inside the electronics, the voltages are analogously processed according to the flow chart shown in Fig. 6.

Essentially the RF voltages $\tilde{U}_{N}$ and $\tilde{U}_{M}$ are subtracted and then as well as $\tilde{U}_{C M}$ frequency filtered. The filtering extracts their fundamental oscillation mode so that the theory of Sec. III can be applied to the remaining, purely sinusoidal voltages. Afterwards the RF voltages are rectified and time averaged to obtain two DC voltages which are proportional to the amplitude of the voltage drop $U_{0}:=\left|\tilde{U}_{N}-\tilde{U}_{M}\right|$ across the circuit and to the amplitude of the current $I_{0}:=\left|\tilde{U}_{C M}\right|$ through the circuit. To assure that the division $I_{0} / U_{0}$ which occurs in Eq. (4) is performed with completely synchronous values of $I_{0}$ and $U_{0}$, which is not guaranteed after the data have been digitized, this process is performed inside of the SCE by an "Analog Computational IC." To obtain the phase shift $\varphi$ between $\tilde{U}_{N}-\tilde{U}_{M}$ and $\tilde{U}_{C M}$ which also occurs in Eq. (4), the amplitudes of both sinusoidal voltages are first aligned. Then an xOR logic generates rectangular pulses of definite height, the broadness of which corresponds to the time delay $\Delta t$ between the zero crossings of $\tilde{U}_{C M}$ and $\tilde{U}_{N}-\tilde{U}_{M}$. The time average (low pass filtering) of this pulse curve finally results in a DC voltage that is proportional to $\Delta t=\varphi / \omega$. Finally, the sinusoidal voltage $\tilde{U}_{C M}$ behind the filter is additionally transformed into TTL pulses which are routed to a frequency counter in the data acquisition unit.

The prototype of the SCE electronics has been developed and manufactured by the DLR Institute of Materials Physics in Space and is currently integrated in the TEMPUS parabolic flight facility. An almost identical, space flight qualified replica of it has by order of the DLR Space Administration been manufactured by the company "Airbus" and was early 2017 integrated into the ISS/EML levitation facility onboard the International Space Station. Since then, several experiments have benefited from this device; see the example in Sec. V.

\section{A. Calibration of the SCE}

It turns out that the relation between each of the three relevant RF input signals $U_{0}=\left|\tilde{U}_{N}-\tilde{U}_{M}\right|, \quad Y_{0}=$ $\left|\tilde{U}_{C M} /\left(\tilde{U}_{N}-\tilde{U}_{M}\right)\right|$, and $\varphi=-\arg \left[\tilde{U}_{C M} /\left(\tilde{U}_{N}-\tilde{U}_{M}\right)\right]$ and the corresponding DC output voltages $U_{N-M}, U_{Y 0}$, and $U_{\varphi}$ can very well be described by the frequency $f$ dependent transfer 


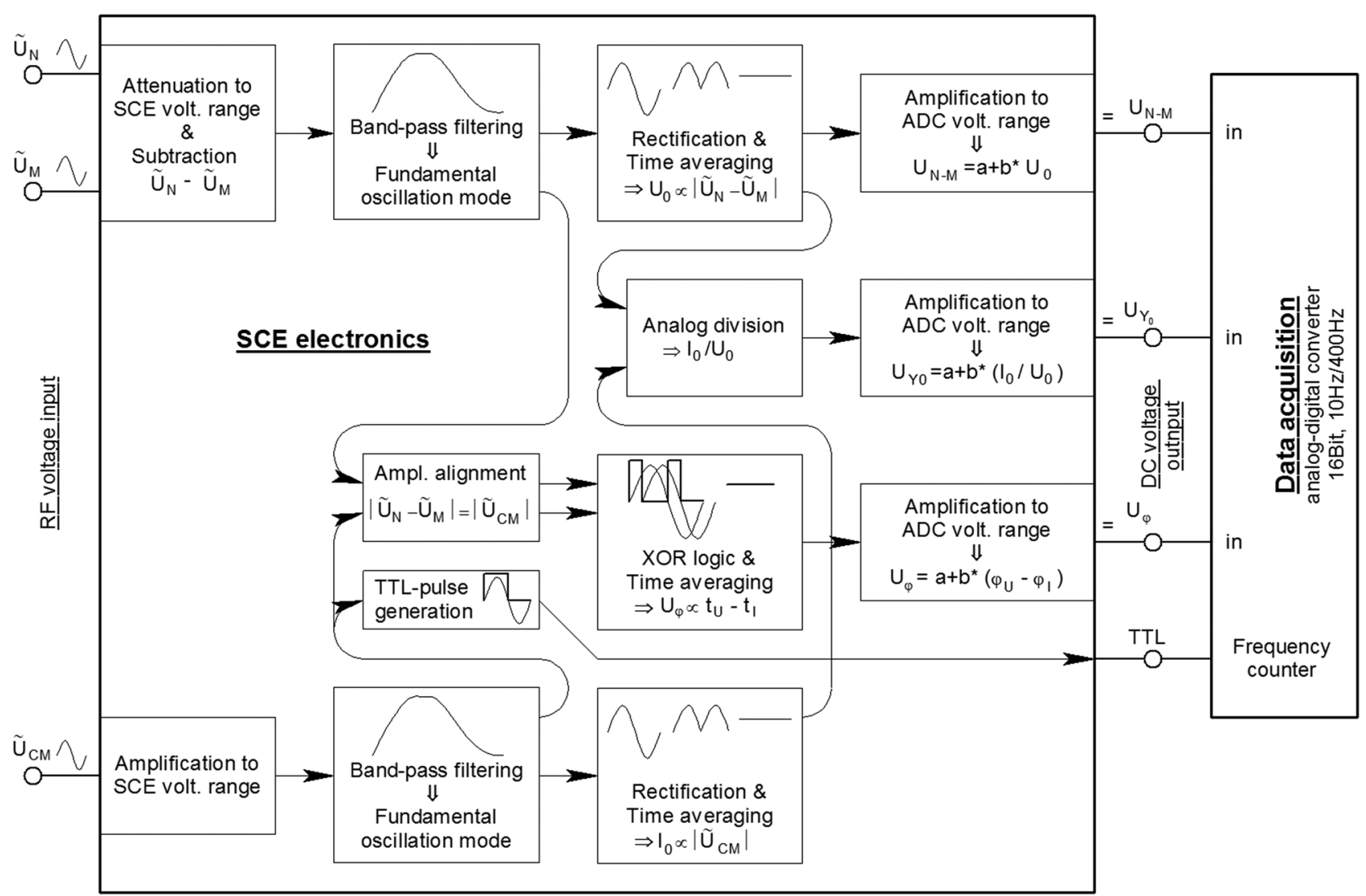

FIG. 6. Flow chart of the SCE measurement electronics modules showing the analog processing of the RF voltages coming from the measurement circuit.

function,

$\mathrm{RFsignal}_{\text {in }}=a+b \cdot\left(1+\sum_{i=1}^{2} \beta_{i} \cdot(f-375 \mathrm{kHz})^{i}\right) \cdot$ DCvolt $_{\text {out }}$.

Calibrations of the SCE electronics, where the RF input signals were generated by a two channel function generator (Tektronix AFG3022) and measured by a RMS voltmeter (Rhode \& Schwartz URE3) and where the corresponding DC output voltages were monitored by the dedicated data acquisition unit (National Instruments Ni USB-6251), show that for appropriate coefficients $a, b, \beta_{1}$, and $\beta_{2}$ in the working range of $\pm 2 \mathrm{kHz}$ around the resonance frequency of the measurement circuit of $375 \mathrm{kHz}$, the relative deviation from this relation is $\left|\Delta U_{0} / U_{0}\right|<2 \times 10^{-4}$ and $\left|\Delta Y_{0} / Y_{0}\right|<2 \times 10^{-4}$ for the first two quantities and that the absolute deviation for the third $\mathrm{RF}$ signal is $|\Delta \varphi|<0.04^{\circ}$. To obtain steady results, the electronics has previously to be powered for at least $2 \mathrm{~h}$ in which essentially the DC-DC power supplies integrated in the SCE heat the device up to an equilibrium temperature of about $37^{\circ} \mathrm{C}$.

\section{MEASUREMENT PROCEDURE AND RESULTS}

Taking the $8 \mathrm{~mm}$ diameter $\mathrm{Zr}_{64} \mathrm{Ni}_{36}$ sample processed in the ISS/EML facility in June 2017 as an example, Fig. 7 shows a typical experiment run valid for both $\mu \mathrm{g}$ electromagnetic levitation facilities.

The electromagnetically positioned $\mathrm{Zr}_{64} \mathrm{Ni}_{36}$ sample is inductively heated, melted, and overheated $(0 \mathrm{~s} \leq t<\approx 6 \mathrm{~s})$.
After the positioning and heating voltages have been reduced, the liquid droplet cools via heat radiation, undercools $\approx 180 \mathrm{~K}$ below its melting temperature, and solidifies where the sudden latent heat release leads to an abrupt temperature increases $(\approx 6 \mathrm{~s}<t<\approx 74 \mathrm{~s})$. After the latent heat is completely emitted, it finally cools down in the solid state $(\approx 95 \mathrm{~s}<t<180 \mathrm{~s})$. While the liquid droplet cools, the output voltages of the SCE $\left(U_{N-M}, U_{Y 0}, U_{\varphi}\right)$ are automatically monitored with an accuracy of $16 \mathrm{Bit}$ and a data rate of $10 \mathrm{~Hz}$ for the ISS/EML facility

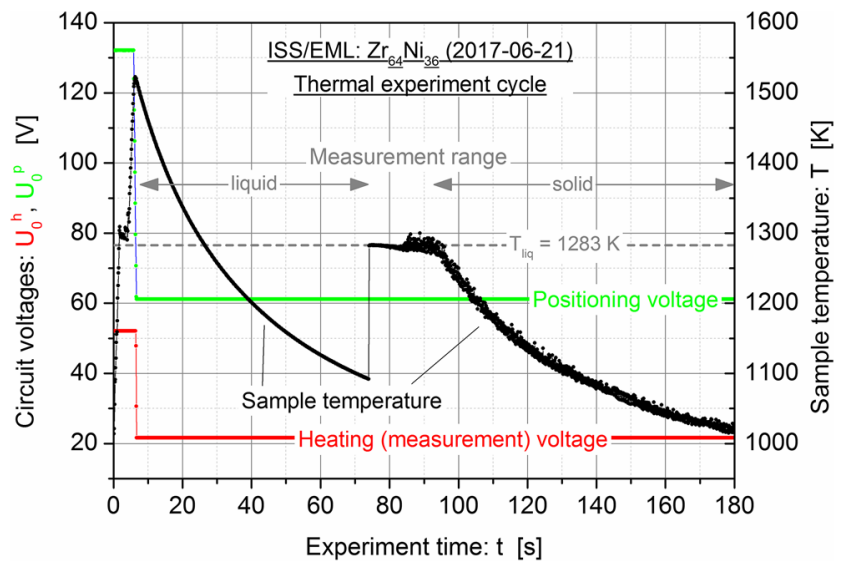

FIG. 7. Example for a typical thermal cycle of an experiment in the ISS/EML or TEMPUS $\mu \mathrm{g}$ levitation facility. Plotted are the voltages of the positioning and heating (measurement) circuit, respectively, as well as the resulting temperature of the processed $\mathrm{Zr}_{64} \mathrm{Ni}_{36}$ sample which was measured by a radiation pyrometer (cf. Fig. 1). 
and of $400 \mathrm{~Hz}$ for the TEMPUS facility. Back transformed via Eq. (17) and together with the simultaneously monitored frequency $f$ and the sample temperature $T$, these data deliver via Eqs. (15), (14), (12), (8), and (7) the temperature dependent electrical resistivity $\rho(T)$ and radius $a(T)$ of the liquid droplet, shown in Fig. 8, after the electrical circuit parameters $R(\langle T w\rangle)$, $C_{H}\left(T_{C}, U_{0}\right)$, and $L$ as well as the coil constant $c c$ have been determined by previous calibration runs.

Especially the processing in the ISS/EML levitation facility onboard the Space Station allows a very quiet, jitter free investigation of the liquid droplet. Besides of the high accuracy of the measurement electronics, see Sec. IV A, and the high data rate $\left(\approx 2 \mathrm{~K}^{-1}\right)$, this is the reason why the resolution of the sample resistivity data $\left\langle\left|\rho-\langle\rho\rangle_{5}\right|\right\rangle_{5} /\langle\rho\rangle_{5}$, shown in the top diagram of Fig. 8 , is $<0.05 \%$ for the whole liquid state. Here, \langle\rangle$_{5}$ means the averaging over 5 neighboring data points. A similar analysis for the resistivity data resulting from the ground based measurement methods described in Refs. 4, 5, 8,9 , and 12 yields resolutions between $\approx 0.5 \%{ }^{4,8}$ and $\approx 2 \%{ }^{9}$ which are an order of magnitude less!

\section{A. Influence of the residual sample deformation}

Measurements are reasonably performed in the cooling phase of the experiment, where the circuit voltages and the resulting magnetic fields are reduced to a minimum; see Fig. 7. This widely avoids fluid flow effects in and magnetic forces

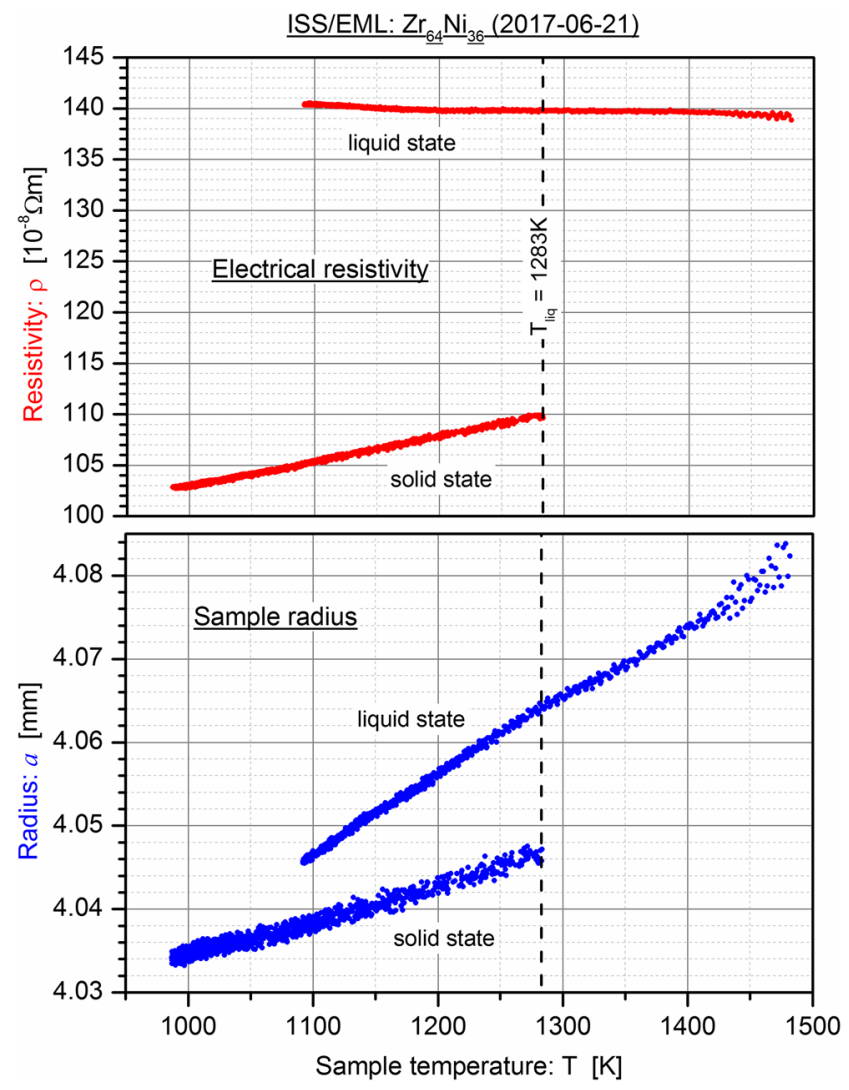

FIG. 8. Using the here processed $\mathrm{Zr}_{64} \mathrm{Ni}_{36}$ sample as example, the top diagram shows its temperature dependent electrical resistivity $\rho(T)$ and the bottom diagram its temperature dependent radius $a(T)$ in the liquid and solid states, respectively. The data were measured with the help of the SCE in the ISS/EML levitation facility onboard the International Space Station. on the liquid sample which otherwise would unduly disturb its assumed perfectly spherical shape caused by the surface tension. Nevertheless, video images from the droplet, recorded during the measurement sidelong through the two coils, see Fig. 2, show a weak prolate ellipsoid with a symmetry axis parallel to the coil axis. Denoting the vertical, long semi-axis of the ellipsoid by $d_{z}$ and the horizontal, short one by $d_{x}$, a detailed image analysis reveals for the relation $d_{x} / d_{z}(T)$ a value of $\approx 0.990$ for the higher sample temperatures $T$ which slightly increases to a value of $\approx 0.992$ for the lower sample temperatures due to the increase in the surface tension.

To quantify the influence of these deformations on the calculation of the sample resistivity, Eq. (13) can be used. It reveals that for a specific measurement value of $\tilde{Z}_{S}(T)$ and $\omega(T)$, the resulting resistivity $\rho(T) \propto a^{2}(T)$ is still proportional to the cross section of the sphere perpendicular to the field lines of the magnetic measurement field, cf. Fig. 2 right. Although derived for a spherical sample, this result can approximately be transposed to the ellipsoid for which the present measurements are performed, i.e., $\rho_{\text {meas }}(T) \propto d_{x}^{2}(T)$. With the condition that the volume of the real ellipsoid and the corresponding ideal sphere are the same, i.e., that $4 / 3 \pi d_{x}^{2} d_{z}=4 / 3 \pi a^{3}$, the relative deviation between measured resistivity and real resistivity adopts the negligibly small value of

$$
\frac{\rho_{\text {meas }}(T)-\rho(T)}{\rho(T)}=\frac{d_{x}^{2}(T)-a^{2}(T)}{a^{2}(T)}=\left(\frac{d_{x}}{d_{z}}\right)^{2 / 3}-1 \approx-0.006,
$$

when the above mentioned measurement values of $d_{x} / d_{z}(T)$ are inserted.

\section{B. Determination of the electrical circuit parameters}

The use of Eqs. (7) and (8) for an evaluation of the experiment data requires the knowledge of the electrical circuit parameters $C_{20}, R_{20}$, and $L$ at $20^{\circ} \mathrm{C}$ as well as of the coefficients $d C / d T$ and $d C / d U_{0}^{2}$, specifying the change of $C_{H}$ with changing capacitor temperature $T_{C}$ and voltage amplitude $U_{0}$, cf. Eq. (6). These parameters can best be obtained by experiment runs without sample, i.e., where $\tilde{Z}_{S}=0$. In this case, Eqs. (7) and (8) reduce under consideration of Eqs. (4)-(6) to

$$
\begin{gathered}
\underbrace{\frac{Y_{0}}{2} \sin (-\varphi) \omega}_{Y I}=\underbrace{\frac{-1}{L}}_{c I 0}+\underbrace{C_{20}}_{c I 1} \cdot \underbrace{\omega^{2}}_{X I 1}+\underbrace{\frac{d C}{d T}}_{c I 2} \\
\cdot \underbrace{\left(T_{C}-20^{\circ} \mathrm{C}\right) \omega^{2}}_{X I 2}+\underbrace{\frac{d C}{d U_{0}^{2}}}_{c I 3} \cdot \underbrace{U_{0}^{2} \omega^{2}}_{X I 3}, \\
\underbrace{\frac{Y_{0} / 2 \cos (-\varphi)(\omega L)^{2}}{1+\alpha_{C u, 20}\left(<T w>-20^{\circ} \mathrm{C}\right)}}_{Y R}=\underbrace{R_{20}}_{c R 0} .
\end{gathered}
$$

For only one definite angular frequency $\omega$, one definite capacitor temperature $T_{C}$ and voltage $U_{0}$, i.e., for only one specified value for each of the known quantities $Y I, X I 1, X I 2, X I 3$, and $Y R$ in Eqs. (19) and (20), the two equations would not permit to determine the five coefficients abbreviated above by $c I_{0}$, $c I_{1}, c I_{2}, c I_{3}$, and $c R_{0}$. The power supply of the measurement circuit allows, however, a variation of the frequency $\omega$ in a 
restricted range. So, together with a variation of the externally controllable circuit voltage $U_{0}$ and of the capacitor temperature $T_{C}$ by powering the circuit, it is possible to create a whole family of Eqs. (19) and (20) with different values for the variables $Y I, X I 1, X I 2, X I 3$, and $Y R$ from which the coefficients $c I_{0}, c I_{1}, c I_{2}, c I_{3}$, as well as $c R_{0}$ can be received by multiple linear regression.

\section{Determination of the coil constant}

To obtain not only the temperature behavior of the sample resistivity $\rho(T)$ and of the sample radius $a(T)$ but also their correct absolute values, the coil constant $c c$ appearing in Eqs. (14) and (15) has to be determined with the help of a spherical calibration sample of well-known electrical resistivity or radius. Very convenient for this is a solid zirconium ( $\mathrm{Zr}$ ) test sphere of known radius $a_{\mathrm{Zr}}(300 \mathrm{~K})$ at room temperature because on the one hand, the resistivity $\rho_{\mathrm{Zr}}(T)$ and thermal expansion $d a_{\mathrm{Zr}} / d T$ of solid $\mathrm{Zr}$ is over a larger temperature range very well-known from the literature ${ }^{27,28}$ and, on the other hand, the solid-solid phase transition of $\mathrm{Zr}$ at $T_{\mathrm{Zr} \text { Phase }}=1148 \mathrm{~K}$ can very well be used for the likewise necessary calibration of the pyrometric sample temperature measurement.

Since a provided sample place especially in the ISS/EML space station facility represents a valuable asset which is reluctantly occupied with a scientifically less attractive zirconium specimen, another, however, less accurate method can also be used to gain $c c$. One of the designated experiment samples which is insensitive to contaminations by mechanical treatment is machined to a sphere of well-known radius $a_{\text {cal }}(300 \mathrm{~K})$ at room temperature. This has been executed, e.g., for the above already discussed $\mathrm{Zr}_{64} \mathrm{Ni}_{36}$ specimen with $a_{\mathrm{ZrNi}}(300 \mathrm{~K})=4.00 \mathrm{~mm}$. If in the solid state $a_{\text {cal }}(T)$ behaves as a function of $T$ linearly in the whole temperature range which is the case for the $\mathrm{Zr}_{64} \mathrm{Ni}_{36}$ sample at least in the upper temperature range, see the bottom diagram of Fig. 8, then the linear extrapolation of the measured radius $a_{c a l}(T \rightarrow 300 \mathrm{~K})$ down to room temperature should result in the machined sample radius at room temperature $a_{c a l}(300 \mathrm{~K})$ provided the coil constant $c c$ already exhibits the correct value. Otherwise $c c$ has to be adjusted correspondingly.

\section{SUMMARY}

The electromagnetic levitation under weightlessness (or microgravity or $\mu \mathrm{g}$ ) provides a very favorable experiment environment for the noninvasive inductive measurement of the electrical resistivity and density of liquid metals. Contrary to standard laboratory conditions, the positioning of a metallic droplet at a definite experiment location under $\mu \mathrm{g}$ can be performed by a symmetric RF magnetic quadrupole field which does no longer inductively interact with the symmetric homogeneous RF magnetic dipole field used for the inductive sample heating and measuring purposes. Furthermore, the forces of the magnetic fields applied on the liquid sample during the inductive measurement process are too low to significantly deform its simple spherical shape.

There are two microgravity electromagnetic levitation facilities which allow the noninvasive inductive measurement of the electrical resistivity and density of liquid metals. The TEMPUS facility operated by the DLR Institute of Materials Physics in Space for short time experiments $(\approx 20 \mathrm{~s})$ during parabolic flights on board of aircrafts and the ISS/EML facility managed by the European Space Agency (ESA) for long time experiments on board the International Space Station (ISS). To enable a high measurement accuracy, both were equipped with a dedicated measurement electronics (SCE).

The preceding sections describe the resistivity measurement setup within the two facilities. The homogeneous magnetic RF dipole field of the levitation facilities, originally used to heat the confined metal samples only, is perfectly suited also for the inductive measurement purposes. This field is generated by a coil which is part of an electrical resonant circuit. The measurement electronics SCE hooked up on this circuit monitors its RF voltages and currents and stores their amplitudes, phase relationships, and frequencies with high resolution and high data rate. The weak damping of the resonant circuit simplifies the theoretical relations between these measurement data and the thermophysical properties, i.e., electrical resistivity and density (radius), of the liquid metallic specimen in the measurement field.

For an evaluation via these theoretical relations, the parameters of the resonant measurement circuit, i.e., inductance, capacitance, and resistance, have to be known. Especially the last two quantities depend sensitively on the temperatures of the capacitor and the coil which have therefore carefully to be measured as well and stored together with the electrical data. The parameters themselves are determined by a calibration run without sample. This "empty coil calibration" would be sufficient if only the relative temperature behavior of sample resistivity and density is of interest. In order to determine also their absolute values, an additional calibration process with a metal sphere (preferably Zirconium) of well-known diameter and resistivity is required.

As proved in the preceding sections, a careful performance of the calibration and measurement process can in the ISS/EML facility result in a quite high resolution of the measured electrical resistivity data in the order of $0.05 \%$ !

\section{ACKNOWLEDGMENTS}

The author gratefully acknowledges the permanent administrative promotion of the SCE project by the DLR Space Administration and the helpful support by his colleagues of the TEMPUS team.

${ }^{1}$ T. Iida and R. I. L. Guthrie, The Physical Properties of Liquid Metals (Clarendon Press, Oxford, 1988).

${ }^{2}$ S. Takeuchi and H. Endo, "Theoretical and experimental studies on the resistivity of molten alloys," Trans. Jpn. Inst. Met. 3, 35-41 (1962).

${ }^{3} \mathrm{~S}$. Takeuchi and H. Endo, "The electrical resistivity of the metals in the molten state," Trans. Jpn. Inst. Met. 3, 30-35 (1962).

${ }^{4}$ T. Richardsen, G. Lohöfer, and I. Egry, "Electrical resistivity of undercooled liquid Cu-Ni alloys," Int. J. Thermophys. 23, 1207-1216 (2002).

${ }^{5}$ B. Delley, H. U. Künzi, and H.-J. Güntherodt, "Contactless resistivity measurements on liquid metals," J. Phys. E: Sci. Instrum. 13, 661-664 (1980).

${ }^{6}$ J. Brillo, G. Lohöfer, F. Schmidt-Hohagen, S. Schneider, and I. Egry, "Thermophysical property measurements of liquid metals by electromagnetic levitation,” Int. J. Mater. Prod. Technol. 26, 247-273 (2006). 
${ }^{7}$ G. Lohöfer and G. Pottlacher, "Inductive measurement of thermophysical properties of electromagnetically levitated metallic melts," High Temp. High Pressures 40, 237-248 (2011).

${ }^{8}$ Y. Ono and T. Yagi, "Electric resistivity of molten Fe-Ni and Fe-Co alloys," Trans. ISIJ 12, 314-316 (1972).

${ }^{9}$ S. G. Teodorescu, R. A. Overfelt, and S. I. Bakhtiyarov, "An inductive technique for electrical conductivity measurements on molten metals," Int. J. Thermophys. 22, 1521-1535 (2001).

${ }^{10}$ W.-K. Rhim and T. Ishikawa, "Noncontact electrical resistivity measurement technique for molten metals," Rev. Sci. Instrum. 69, 3628-3633 (1998).

${ }^{11}$ J. E. Enderby, S. Ansell, S. Krishnan, D. L. Price, and M.-L. Saboungi, "The electrical conductivity of levitated liquids," Appl. Phys. Lett. 71, 116-118 (1997).

${ }^{12}$ L. Skinner and A. C. Barnes, "An oscillating coil system for contactless electrical conductivity measurements of aerodynamically levitated melts," Rev. Sci. Instrum. 77, 123904 (2006).

${ }^{13}$ G. E. Rustan, N. S. Spyrison, A. Kreyssig, R. Prozorov, and A. I. Goldman, "Noncontact technique for measuring the electrical resistivity and magnetic susceptibility of electrostatically levitated materials," Rev. Sci. Instrum. 83, 103907 (2012).

${ }^{14} \mathrm{G}$. Lohöfer, "Electrical resistivity measurement of liquid metals," Meas. Sci. Technol. 16, 417-425 (2005).

${ }^{15}$ E. C. Okress, D. M. Wroughton, G. Comenetz, P. H. Brace, and J.C. R. Kelly, "Electromagnetic levitation of solid and molten metals," J. Appl. Phys. 23, 545-552 (1952).

${ }^{16} \mathrm{G}$. Lohöfer, "Theory of an electromagnetically levitated metal sphere," SIAM J. Appl. Math. 49, 567-581 (1989).

${ }^{17}$ A. Seidel, W. Soellner, and C. Stenzel, "EML-An electromagnetic levitator for the International Space Station,” J. Phys.: Conf. Ser. 327, 012057 (2011).
${ }^{18}$ I. Egry, H. Giffard, and S. Schneider, "The oscillating drop technique revisited," Meas. Sci. Technol. 16, 426-431 (2005).

${ }^{19}$ R. K. Wunderlich and H.-J. Fecht, "Modulated electromagnetic induction calorimetry of reactive metallic liquids," Meas. Sci. Technol. 16, 402-416 (2005).

${ }^{20} \mathrm{G}$. Lohöfer and I. Egry, "Electrical resistivity measurement in TEMPUS: Results for solid, liquid and undercooled liquid $\mathrm{Co}_{80} \mathrm{Pd}_{20}$," in Solidification 1999, edited by W. H. Hofmeister, J. R. Rogers, N. B. Singh, S. P. Marsh, and P. W. Vorhees (The Minerals, Metals \& Materials Society, Warrendale, 1999), pp. 65-74.

${ }^{21}$ R. K. Wunderlich and H.-J. Fecht, "Thermophysical properties of bulk metallic glass forming alloys in the stable and undercooled liquid-A microgravity investigation," Mater. Trans. 42, 565-578 (2001).

${ }^{22} \mathrm{P}$. R. Rony, "The electromagnetic levitation of metals," in Proceedings of Transactions Vacuum Metallurgy Conference 1964, edited by M. A. Cocca (American Vacuum Society, Boston, MA, 1965), pp. 55-135.

${ }^{23}$ V. Bojarevics and K. Pericleous, "Modelling electromagnetically levitated liquid droplet oscillations," ISIJ Int. 43, 890-898 (2003).

${ }^{24}$ R. W. Hyers, "Fluid flow effects in levitated droplets," Meas. Sci. Technol. 16, 394-401 (2005).

${ }^{25} \mathrm{G}$. Lohöfer, German patent 3836239 (08 August 1991); U.S. patent 4,993,043 (12 February 1991).

${ }^{26} \mathrm{G}$. Lohöfer, "Magnetization and impedance of an inductively coupled metal sphere," Int. J. Eng. Sci. 32, 107-117 (1994).

${ }^{27}$ N. D. Milosevic and K. D. Maglic, "Thermophysical properties of solid phase Zirconium at high temperatures," Int. J. Thermophys. 27, 1140-1159 (2006).

${ }^{28}$ V. Petukhov, "Thermal expansion of Zirconium in the solid phase," High Temp. - High Pressures 35-36, 15-23 (2003). 